$85 \%$ had more than 4 risk factors, the most common: NICU admission(100\%), central catheter(100\%), parenteral nutrition $(93 \%)$, broad-spectrum ATB use(86\%) and $\operatorname{IMV}(71 \%)$. The most frequent associated pathology was catheter-related infection(43\%) and necrotizing enterocolitis(22\%). No CNS involvement was identified in any case.

Conclusions Systemic prophylaxis with fluconazole has been an effective measure for the reduction of invasive fungal infection in our unit, with a decrease between $40-70 \%$. However, optimization of this strategy is necessary, focusing on those at highest risk $(<1000 \mathrm{~g}$ and/or $\leq 27$ weeks).

\section{A 7-YEARS RETROSPECTIVE STUDY OF NOSOCOMIAL CANDIDA INFECTION IN TERTIALLY NICU}

doi:10.1136/archdischild-2012-302724.1172

U Godula-Stuglik, J Balicz, MWB Kwiatkowska-Gruca, A Pukas. Department of Pediatrics, NICU, Zabrze, Poland

Background Nosocomial Candida infections (NCI) with dominant C. albicans account for $6-18 \%$ of lateonset sepsis in NICU, with mortality rate $22-32 \%$ and increase health care costs.

Aim Evaluation morbility and mortality rate of neonatal NCI, considering sex, GA, BW, perinatal risk factors, occurence of other diseases, types of Candida, number of NCI episodes.

Material and Methods The analysis involved 70 newborns (41 boys, 29 girls), 27 ELBW, 20 VLBW, 11 LBW and $12>2.5 \mathrm{~kg}$, treated wihin $2002-7$ years (4.2\% of all), all with flukonazole prophylaxis. Mycological examination was based on Sabouroud medium and using Vitek 2 apparatus.

Results 103 cases of NCI (46 single, 4 double, $7^{3} 3$ ) were diagnosed between 8 and 117 day of hospitalization $\left(27 \% £ 15^{\text {th }}, 32 \%\right.$ between $16^{\text {th }}$ and $30^{\text {th }}, 41 \%>30^{\text {th }}$ day). Eighteen types of $\mathrm{C}$. were isolated (44\% in blood), most often albicans $(26 \%)$, sake $(25 \%)$ and lusitaniae $(18 \%)$. The significant dependence was stated between newbons' death and their GA and number of C. episodes. Presence of central catheters, $\mathrm{MV}$, bacterial sepsis and ventilator associated pneumonia, total parenteral nutrition and severe RDS, BPD, IVH, NEC were founded as major risk factors for neonatal NCI.

\section{Conclusions}

1. Fetal maturity and number of NCI episodes determine the prognosis in newborns infected due to Candida.

2. Risk factors must be evaluated carefully in all sick newborns, because of longer NICU stay and necessity of invasive procedures.

\section{BURKHOLDERIA GLADIOLI SEPSIS IN NEWBORNS}

doi:10.1136/archdischild-2012-302724.1173

'A Dursun, ${ }^{1} \mathrm{~A}$ Zenciroglu, ${ }^{1} \mathrm{BS}$ Karagol, ${ }^{1} \mathrm{H}$ Nilay, ${ }^{1} \mathrm{~N}$ Okumus, ${ }^{1} \mathrm{~S}$ Beken, ${ }^{2} \mathrm{~N}$ Gol, ${ }^{3} \mathrm{G}$ Tanir. ${ }^{1}$ Neonatology; ${ }^{2}$ Microbiology; ${ }^{3}$ Pediatric Infectious Diseases, Dr. Sami Ulus Maternity and Children's Health and Diseases Training and Research Hospital, Ankara, Turkey

Background and Aim Burkholderia gladioli is a rare cause of bacteraemia and sepsis in patients without predisposing factors like chronic granulomatous disease, cystic fibrosis or immunsupressive disorders. There is little known about $B$. gladioli infections in newborns. The aim of this study was to evaluate the features of $B$. gladioli infections in newborns.

Methods Clinico-pathologic characteristics, patterns of antimicrobial susceptibility, predisposing factors and outcomes of $B$. gladioli bloodstream infections of newborn patients were analysed retrospectively from 2008 to 2011.

Results During the 3 -year study period, B. gladioli was isolated from blood cultures of 14 patients (3.7 per 1000 admissions). Five out of $14(35.7 \%)$ cases have a positive blood culture at the time of initial admission. Primary diagnoses of neonates were severe major congenital anomalies, congenital leukemia, prematurity with respiratory distress syndrome, pneumonia and parapneumonic pleural effusion. Eleven of the 14 patients (78.6\%) had undergone at least one invasive procedure and $71.4 \%$ of the patients had undergone two or more of invasive procedures. The most susceptible antimicrobial agents were amikacin, gentamicin, imipenem, ciprofloxacin, trimethoprime/sulphametaxazole and ceftriaxone. The overall inhospital mortality rate was $21.4 \%$. The mortality rate was $7 \%$ for $B$. gladioli infections.

Conclusions $B$. gladioli might be a causative microorganism of both early neonatal and nosocomial sepsis in newborns. To our knowledge, this is the first report of B. gladioli infection in newborns. Although it seems to have a low pathogenic potential and insidious clinical course in newborns, resistance patterns to antibiotics may be a problem. Mortality was mainly associated with underlying diseases.

\section{SEPSIS AMONG PRETERM INFANTS WITH BIRTH WEIGHT $>750$ G: EXPERIENCE OF A MEDICAL CENTER IN NORTHERN TAIWAN}

doi:10.1136/archdischild-2012-302724.1174

1,2MC Chiang, ' $O W$ Chan, ${ }^{1} Y L$ Chau, 1,2JJ Lin, 'R Lien, 'PH Yang. 'Pediatrics, Chang Gung Memorial Hospital; ' ${ }^{2}$ Graduate Institute of Clinical Medical Sciences, Chang Gung University College of Medicine, Taoyuan, Taiwan R.O.C.

Background Sepsis is a major cause leading to neonatal mortality and morbidity, particularly for tiny preemies. The purpose of this study aimed to compare outcome between infants with birth weight $(\mathrm{BBW}) \leq 750 \mathrm{~g}$ having culture-positive sepsis and infants without any positive culture.

Methods This was a retrospective cohort study of infants with BBW $\leq 750$ g admitted to Chang Gung Children's Hospital between January 2006 and December 2010. Sepsis was defined as infants had clinical signs and positive blood culture results. Outcome, pathogens and clinical data were collected.

Results 154 infants were enrolled; the gestational age (GA) and BBW were $25.1 \pm 1.9$ weeks and $639.6 \pm 88.5 \mathrm{~g}$ (mean \pm SD), respectively. 46 patients $(29.9 \%$ ) had sepsis and the incidence of sepsis was 5.2 episodes per 1000 patient days. There were 62 episodes of sepsis involving 66 pathogens during the study period. 38 gram-positive pathogens (57.6\%), 22 gram-negative pathogens (33.3\%) and 6 fungal infection $(9.1 \%)$ were identified. The major causative pathogens were coagulase negative staphylococcus $(n=24)$, Escherichia coli $(n=7)$ and klebsiella pneumoniae $(n=7)$. Infants received patent ductus arteriosus ligation or had retinopathy of prematurity requiring therapy were associated with developing sepsis thereafter. There was no significant difference in GA, BBW, gender, Apgar scores, intraventricular hemorrhage, bronchopulmonary dysplasia and mortality between sepsis and non-sepsis groups. The mortality rate was $42.9 \%$, and sepsis related mortality accounted for $14.5 \%$ of mortality in the current study.

Conclusions One third of infants with BBW $\leq 750 \mathrm{~g}$ had sepsis. Based on the finding of identified pathogens, nosocomial infection was still the major cause for sepsis.

\section{PATHOGENS WHICH CAUSING NEONATAL INFECTION IN MECONIUM STAINED AMINIOTIC FLUIDS}

doi:10.1136/archdischild-2012-302724.1175

M Kosim, A Eka Rini, G Irawan Sarosa, A Nur Radityo. Neonatology Division, Department of Pediatrics, Faculty of Medicine, Diponegoro University/Dr. Kariadi Hospital, Semarang, Indonesia

Background Few studies considered that amniotic fluid is sterile but some others mentioned that contains pathogens. Even though not all meconium stained amniotic fluids MSAF develop into 